\title{
L'Umanesimo italiano. Problemi e studi di ieri e di oggi
}

\section{Riccardo Fubini}

\section{Q OpenEdition \\ 9 Journals}

\section{Edizione digitale}

URL: http://journals.openedition.org/studifrancesi/9395

DOI: ERREUR PDO dans /localdata/www-bin/Core/Core/Db/Db.class.php L.34 : SQLSTATE[HYO00]

[2006] MySQL server has gone away

ISSN: 2421-5856

\section{Editore}

Rosenberg \& Sellier

\section{Edizione cartacea}

Data di pubblicazione: 1 décembre 2007

Paginazione: 504-515

ISSN: 0039-2944

Notizia bibliografica digitale

Riccardo Fubini, «L'Umanesimo italiano. Problemi e studi di ieri e di oggi», Studi Francesi [Online], 153 (LI | III) | 2007, online dal 30 novembre 2015, consultato il 07 janvier 2021. URL: http://

journals.openedition.org/studifrancesi/9395 ; DOI: https://doi.org/10.4000/studifrancesi.9395

\section{(c) (i) (9)}

Studi Francesi è distribuita con Licenza Creative Commons Attribuzione - Non commerciale - Non opere derivate 4.0 Internazionale. 


\section{L'Umanesimo italiano Problemi e studi di ieri e di oggi}

È di recente uscita una raccolta di saggi sintetici, in ambito internazionale, sulle «interpretazioni dell'Umanesimo rinascimentale». Il curatore, Angelo Mazzocco, confessa in sede introduttiva di non saper dare una sintesi delle prospettive critiche dei vari contributi, in considerazione della loro eterogeneità, tale da rendere arduo anche solo il delineare i termini della discussione ${ }^{2}$. Tale è infatti, come si suol dire, lo stato della questione dopo la recente scomparsa delle personalità che più avevano esercitato influenza nelle sedi disciplinari intercomunicanti degli studi sull'Umanesimo, quali i filosofi Eugenio Garin e Paul Oskar Kristeller, lo storico Hans Baron (alla memoria dei quali è dedicato il volume ora citato del Mazzocco), a cui vanno aggiunti, per quanto riguarda l'Italia, i filologi Alessandro Perosa, Giuseppe Billanovich e Augusto Campana, gli italianisti Carlo Dionisotti e Vittore Branca, per non dire della memoria più lontana di Franco Simone, che il presente convegno ha voluto onorare.

Diversamente dall'amico Mazzocco, ritengo sia giunta l'ora di trarre un bilancio, che doverosamente coincida con un ripensamento critico, e con esso, mi auguro, rinnovate direttive metodiche e programmatiche. La sede degli studi rinascimentali - e il presente convegno ne è ulteriore conferma - è ovviamente quella internazionale, al di là del rispetto, pur doveroso, delle specifiche tradizioni nazionali.

Lo studioso italiano che faccia, come chi scrive, professione di storico trova scarso riscontro, per quel che riguardi il campo di studio dell'Umanesimo quattrocentesco, nella tradizione patria ${ }^{3}$. Tale vera e propria lacuna ha radici profonde nella storia, di cui si possono segnalare due momenti essenziali. Il primo, più lontano e intrinseco alla cultura rinascimentale stessa, è la mancata trasmissione a stampa (e cioè in pubblica sede) della più significativa produzione umanistica quattrocentesca, alle scaturigini di quel movimento di cultura. A cominciare dal Petrarca latino, i maggiori fra gli umanisti, da Poggio Bracciolini a Lorenzo Valla, da Enea Silvio Piccolomini a Marsilio Ficino (per non parlare di Leonardo Bruni, che fu una riscoperta dell'erudizione sette-ottocentesca, o, peggio ancora, di Leon Battista Alberti, organicamente edito solo nel nostro tempo), ebbero le loro opere pubblicate, dopo le edizioni incunabolo, esclusivamente in sede transalpina, a Parigi, Strasburgo, Colonia, Basilea. In altri termini la loro pubblica diffusione, non importa se manoscritta o a stampa, resse fin quando ressero con sovranità riconosciuta gli Stati italiani indipendenti. Calò poi il sipario di una censura implicita, ben prima di quella dichiarata con il formalizzarsi

(1) Interpretations of Renaissance bumanism, ed. by A. Mazzocco, Leiden 2006.

(2) A. Mazzocco, Introduction, ivi, pp. 1-18, qui p. 17: «The interpretations of Renaissance humanism provided by the scholars included in this volume, like those of their predecessors in the nineteenth and twentieth centuries, are varied to the point of being contradictory». Si veda anche al riguardo Palgrave advances in Renaissance bistoriography, ed. by J. WoOLFson, New York, Houndmills 2005 .

(3) Mi sia lecito premettere le raccolte dei miei principali contributi: R. FUBINI, Umanesimo e secolarizzazione da Petrarca a Valla, Roma 1990; ID., L'umanesimo italiano e $i$ suoi storici. Origini rinascimentali - critica moderna, Milano 2001; ID., Storiografia dell'umanesimo in Italia da Leonardo Bruni ad Annio da Viterbo, Roma 2003. 
della congregazione dell'Indice4. Nella fioritura culturale del xvi secolo le tradizioni umanistiche furono tendenzialmente ridotte a classicismo letterario, e cioè disciplinate secondo un risorgente criterio di autorità; ovvero, fatto ancor più insidioso, furono sostituite da nuovi testi di argomento affine, ma ispirate o a stretto specialismo di scuola, o ai più neutri criteri della compilazione enciclopedica. Per limitarsi a due esempi significativi, al razionalismo empirico, che in sede grammaticale e linguistica aveva ispirato le Elegantiae latinae linguae di Lorenzo Valla, subentrarono i dettami retorici del ciceronianesimo5; per altro riguardo la considerazione critica del mutamento storico, che aveva informato la descrizione corografica dell'Italia illustrata di Biondo Flavio (1453), viene sostituita, giusto un secolo dopo, dalla Descrittione di tutta l'Italia del domenicano Leandro Alberti (1568), che si guarda bene dal riconoscere il debito al predecessore, e indulge, al contrario di lui, alla compilazione e alla leggenda ${ }^{6}$. Così uno degli ultimi eminenti eredi della cultura critica dell'Umanesimo, Carlo Sigonio scoraggiava nel 1559 i giovani transalpini che ancora affollavano le università italiane: essi in realtà non si accorgevano che «humanitatem illam [...], quae propria quondam Italiae fuisset, nunc demum nimia nostrorum facilitate, ne an ignavia hominum dicam, finibus nostris emissam, ad vos se et vestri similes quasi in colonias nobilissimas emigrasse» ${ }^{7}$. In altre parole, la prima tradizione umanistica non apparteneva più, e non sarebbe più appartenuta ai termini stretti della storia nazionale.

L'altro episodio, non meno significativo, è molto più recente. Sulla fine del 1868 usciva a Lipsia la seconda edizione riveduta del Die Kultur der Renaissance in Italien di Jacob Burckhardt (quella originaria era del 1860), e di qui partì la fortuna europea di quest'opera, che, facendo incominciare l'epoca della 'modernità' dal Rinascimento italiano, apriva una discussione che, specie in seno alla Kulturgeschichte tedesca, si sarebbe protratta per oltre mezzo secolo. Ma in Italia essa incontrò, anche in considerazione del progetto in corso di una traduzione, l'opposizione risoluta di Francesco De Sanctis. L'immagine ambivalente della «modernità» proposta da Burckhardt (un'apertura fin qui inaudita alle conoscenze, all'azione e all'arte, non accompagnata però dalle vecchie, non sostituite certezze morali) non apparteneva, né sarebbe più dovuta appartenere alla giovane nazione ormai unificata, volta al proprio riscatto. Tale è il senso preciso del celebre saggio, L'uomo del Guicciardini, scritto per l'appunto nel 1869, che così descrive (e rovescia) l'immagine burckhardtiana, quale una «civiltà», che «toccava quell'ultima perfezione che si manifestava nel lusso e nell'eleganza», ma che in ciò stesso denotava il proprio decadimento. Sicché, secondo De Sanctis, in ovvio riferimento a Burckhardt, $i$ «giudizi degli storici oltremontani» andavano mantenuti estranei alla storia nazionale, come egli stesso dimostrava eloquentemente nella forte impronta moralistica con cui, nella pressoché coeva Storia della letteratura italiana, trattò del «secolo chiamato del risorgimento, e che fu pur quello della nostra decadenza» ${ }^{8}$. La conseguenza fu che le tematiche burckhardtiane, pur così dibattute

(4) Cfr. R. Fubini, Pubblicità e controllo del libro nella cultura del Rinascimento. Censura palese e condizionamenti coperti dell'opera letteraria dal tempo del Petrarca a quello del Valla, in Humanisme et Église en Italie et en France méridionale ( $X V^{*}$ sièclemilieu $d u X V I^{e}$ ), sous la direction de P. GILLI, Roma, École française de Rome, 2004, pp. 201-237.

(5) Cfr. V. DE CAPrio, Le "Elegantiae" di Lorenzo Valla, in Letteratura italiana. Le opere. Vol. I: Dalle origini al Cinquecento, Torino 1992, pp. 647-679.

(6) Cfr. R. Fubini, Note su Leandro Alberti e l' Italia illustrata" di Biondo Flavio, in L'Italia dell'Inquisitore. Storia e geografia dell'Italia del Cinquecento nella "Descrittione" di Leandro Alberti, a cura di M. DonatTini, Bologna 2007, pp. 137-143.

(7) Cfr. W. McCuaig, Andreas Patricius, Carlo Sigonio, Onofrio Panvinio, and the Polish nation of the University of Padua, «History of Universities», 3 (1983), p. 93.

(8) Cfr. R. Fubini, L'uomo del Guicciardini tra De Sanctis e Burckhardt, in ID., L'umanesimo italiano, cit., pp. 230-238. 
nell'ambito della cultura europea, furono pressoché ignorate in sede nazionale. Quivi prevalsero l'erudizione e l'aneddotica letteraria, mentre, negli studi più specifici sull'Umanesimo latino, si affermò la filologia di studiosi dell'antichità, a cominciare dal benemerito latinista Remigio Sabbadini; mentre Benedetto Croce, trascurando l'Umanesimo delle origini, rivolse piuttosto la sua attenzione agli autori «del pieno e del tardo Rinascimento», a partire dalla storia napoletana di età aragonese?.

La sola via che, sulla scorta della rivendicazione nazionale di un 'primato' sulla via della modernità, si riallacciò alle tematiche più ampiamente filosofico-culturali dell'età del Rinascimento, fu quella indicata dalla scuola neo-hegeliana, che a partire da Bertrando Spaventa conduce soprattutto a Giovanni Gentile, in un'improbabile combinazione degli assunti anti-finalistici del disincantato Burckhardt, con il teleologismo, da lui deprecato, di matrice hegelian $a^{10}$. Sicché a tener banco in materia, fino circa agli anni del dopoguerra, furono da un lato le speculazioni ancora d'impronta gentiliana, e, dall'altro, come si è detto, la filologia e l'erudizione, che, detto per inciso, si stavano prendendo la loro rivincita sulla critica letteraria di matrice desanctisiana e crociana, vicina ormai al suo esaurimento. Viceversa, per quanto riguarda la storiografia, come eloquentemente denunciava Federico Chabod a proposito appunto della storia rinascimentale, gli studi «di ordine propriamente politico» e quelli «di ordine culturale» avevano teso a «rinchiudersi in una sfera propria e autonoma, a cui nessun o assai fioco raggio di luce perviene alla vicina» ${ }^{11}$.

$\mathrm{Ma}$ è agli studi di storia filosofica e culturale a cui dobbiamo ancora rivolgere la nostra attenzione. A subentrare all'ormai declinante gentilianesimo fu la figura, ricca d'estro e sensibilità culturale, ma altrettanto difficile e sfuggente di Eugenio Garin ${ }^{12}$. Autore, nel 1937, di una monografia, improntata al clima dello spiritualismo idealistico, su Pico della Mirandola ${ }^{13}$, fu quindi curatore di raccolte di testi umanistici allora pressoché dimenticati o dispersi, da Pico stesso a Coluccio Salutati, dalla silloge dei Filosofi italiani del Quattrocento ${ }^{14}$, a un compendio illustrato di testi, Il Rinascimento italiano $^{15}$, per poi dare una veste ultima a questo genere congeniale di fatiche con la raccolta, ormai negli anni del dopoguerra, dei Prosatori latini del Quattrocento ${ }^{16}$. È ora notevole che, pur con tutte le sfumature che la varia gamma dei testi suggerisce, stentino ad affermarsi figure individuali (difetto del resto comune al paradigma, duro a morire, dell' "umanista"), e d'altro canto ancora sostanzialmente prevalga il tema spiritualistico, caro a Gentile, della «dignità dell'uomo» ${ }^{17}$. «Trionfo dell'umana spiritualità», è detta l'epoca del Rinascimento in genere $^{18}$; e tale tema percorre anche

(9) Cfr. B. Croce, Poeti e scrittori del pieno e del tardo Rinascimento, voll. 2, Bari 1945.

(10) Cfr. G. GENTILE, Il pensiero italiano del Rinascimento, $4^{\mathrm{a}}$ edizione, Firenze 1968 (l'edizione originale della raccolta è del 1937). Il capitolo più significativo, il terzo, Il concetto dell'uomo nel Rinascimento, risale al 1916. Sull'interpretazione del Burckhardt, cfr. il mio Origini e significato del "Die Kultur der Renaissance in Italien" di Jacob Burckhardt, in R. FubINI, L'umanesimo italiano, cit., pp. 211-229.

(11) F. Chabod, Gli studi di storia del Rinascimento, in Cinquant'anni di vita intellettuale italiana, 1896-1946, a cura di C. ANTONI e R. MatTioli, Napoli 1950, vol. I, pp. 126-207, qui p. 128; ora anche in ID., Scritti sul Rinascimento, Torino 1967, pp. 147-148.

(12) Si cfr. in primo luogo Bibliografia degli scritti di Eugenio Garin, Bari 1969 e E. GARIN, La filosofia come sapere storico, Bari 1990; ID., Intervista sull'in- tellettuale, a cura di M. AIELlo, Roma-Bari 1997; e anche M. CAPATI, Cantimori, Contini, Garin: Crisi di una cultura idealistica, Bologna 1997, pp. 73-105.

(13) E. Garin, Giovanni Pico della Mirandola. Vita e dottrina, Firenze 1937.

(14) Filosofi italiani del Quattrocento, pagine scelte, tradotte e illustrate da E. GARIN, Firenze 1942 («Pubblicazioni dell'Istituto Nazionale di Studi sul Rinascimento»).

(15) E. Garin, Il Rinascimento italiano, Milano 1941 (Istituto per gli Studi della Politica Internazionale).

(16) Prosatori latini del Quattrocento, a cura di E. GARIN, Milano-Napoli 1952 («La Letteratura italiana. Storia e testi»).

(17) Si veda in particolare al riguardo E. GARIN, La "Dignitas hominis" e la letteratura patristica, in «La Rinascita», I (1938), pp. 102-146.

(18) Cfr. E. Garin, Il Rinascimento, cit., p. 11. 
la Storia della filosofia, scritta fra il 1940 e il 1942 per la «Storia dei generi letterari italiani» della Vallardi, a sostituzione di quella lasciata incompiuta da Gentile, e pubblicata solo nel $1947^{19}$. In quello stesso anno usciva in veste tedesca a Berna il volume di sintesi che più avrebbe dato fama all'autore, L'Umanesimo italiano. Filosofia e vita civile nel Rinascimento ${ }^{20}$. Come risulta fin dall'intitolazione, qui per la prima volta compare, in primo piano almeno, il tema dell'«Umanesimo civile», di qui intendendo quello del rapporto dell' umanista' con la società e la politica, e di qui dell'apprezzamento della 'vita activa' e dei beni terreni nell'ambito di un regime repubblicano; ideali questi ultimi che sarebbero decaduti nella seconda metà del secolo, in coincidenza con l'avvento della signoria medicea e il ritorno, con la filosofia neoplatonica, al primato della contemplazione metafisica: «Se il primo Umanesimo fu tutto un'esaltazione della vita civile, della libera costruzione umana di una città terrena, la fine del '400 è caratterizzata da un chiaro orientamento verso un'evasione dal mondo, verso la contemplazione» ${ }^{21}$.

Non mi soffermo a discutere se tali tematiche, o perlomeno l'enfasi posta su di esse, fossero suggerite al sempre percettivo Garin dalle ricerche parallele di Hans Baron, che almeno fin dagli anni ' 30 aveva insistito sul legame intrinseco fra il primo Umanesimo fiorentino e «la rinascita dell'etica statale romana», come parte di un progetto di lavoro su «Lo spirito comunale dell'Umanesimo fiorentino secondo la 'Vita civile' di Matteo Palmieri e altre fonti» ${ }^{22}$, che si sarebbe poi tramutato, nel dopoguerra in terra d'America, in The Crisis of the Early Italian Renaissance del 1955. Di Baron si dirà poi. Né è qui luogo di speculare (ché poco importa dal punto di vista dell'interpretazione storica) se già a questa data alta fosse sensibile in Garin, come poi dichiaratamente nelle Cronache della filosofia italiana (1955), l'ideologizzazione gramsciana dell' «intellettuale organico» e l'idea corrispettiva della «cultura impegnata» ${ }^{23}$. In fondo il convergere del pensiero nell'azione (propugnato da Gramsci con una precisa connotazione politica) non si discostava, in linea teorica almeno, dall'attualismo gentiliano, per non dire di altre dottrine e tradizioni di pensiero, a cominciare dal molto germanico 'consenso allo Stato', quale obbligazione imprescindibile del cittadino e del dotto, a cui - molto più di quanto sia stato generalmente avvertito - fece capo il Baron nel formulare le sue 'tesi' sull' Umanesimo civile' fiorentino ${ }^{24}$. Infine,

(19) E. Garin, La filosofia. I, Dal Medioevo all'Umanesimo; II, Dal Rinascimento al Risorgimento, Milano 1947. Cito dalla seconda edizione ampliata, Storia della filosofia italiana, Torino 1966, vol. I, p. XIII. Si veda per esempio ivi, p. 227, a proposito dell'interpretazione millenaristica del Rinascimento di K. Burdach: «E certo difficile non avvicinare Cola di Rienzo a Petrarca, ed è altrettanto non tenere conto dell'ansia rinnovatrice del tribuno romano»; e anche il capitolo III, Il mondo degli nomini, a proposito di Giannozzo Manetti: «Già nel Manetti si mostra chiara la sempre maggiore presenza, accanto all'esaltazione della vita attiva, terrena, civile, di motivi stoici e neoplatonici, volti a sottolineare il valore dello spirito umano nel suo significato metafisico» (p. 334). Notevole anche l'affermazione su L.B. Alberti, che lo stesso Garin avrebbe provveduto a capovolgere in saggi a noi più vicini: «L'inno alla dignità dell'uomo, sciolto nel secondo libro della Famiglia, esprime veramente uno dei grandi temi di tutta l'opera dell'Alberti» (p. 348). Si cfr. qui oltre, n. 41.

(20) E. GARIN, Der italienische Humanismus. Philosophie und bürgerliches Leben in Renaissance, nach dem Manuscript ins Deutsche bertragen von G. ZAmBOnI, Bern 1947.

(21) ID., L'Umanesimo italiano. Filosofia e vita civile nel Rinascimento, Bari 1952, p. 103. Nell'Introduzione aggiunta per l'edizione italiana, l'A. insiste su di un altro tema ancora, il valore della nuova sensibilità storica acquisita dagli umanisti, che «permise di stabilire la nostra distanza rispetto a quel passato», e sul significato di qui assunto dalla «filologia» (p. 23). In un Epilogo parimenti aggiunto, allo «schema di una filosofia teologizzante» viene contrapposta la «scienza dell'universale», che «vive come coscienza di sé in ogni concreta ricerca» (p. 278).

(22) Si veda al riguardo E. GARIN, Le prime ricerche di Hans Baron sul Quattrocento e la loro influenza tra le due guerre, in Renaissance studies in honor of Hans Baron, ed. by A. MolHo and J.A. TeDESCHI, Firenze 1971, pp. LIX-LXX, in particolare p. LXVI.

(23) Cfr. E. Garin, Cronache di filosofia italiana (1900-1943), Bari 1955 (seconda edizione definitiva, ivi 1959).

(24) Cfr. il mio saggio, Una carriera di storico del Rinascimento: Hans Baron, in R. FUBINI, L'Umanesimo italiano, cit., pp. 277-316. 
nel riprendere in mano L'Umanesimo italiano di Garin, ci si sorprende a constatare come il modello complessivo dell'opera sia ancora conforme alla prospettiva storica di Gentile, che accomunava 'Umanesimo' e 'Rinascimento' in un unico complesso epocale: «L'Umanesimo - scriveva - è la preparazione, o, se si vuole, l'inizio del Rinascimento» ${ }^{25}$. Sicché anche il volume sintetico di Garin è equamente ripartito fra Quattro- e Cinquecento, e, prendendo le mosse da Petrarca e Salutati, termina con la triade di Telesio, Bruno e Campanella, con buona pace degli umanisti 'civili' fiorentini del primo Quattrocento.

Ma l'Umanesimo civile di Garin è altresì significativo per un'altra, per dir così, meno campanilistica questione. Come si è detto, esso apparve in prima edizione in veste tedesca a Berna nel 1947. Il fatto insolito cela un risvolto di notevole interesse, per quanto sottaciuto dall'autore nell'edizione italiana del 1952. Promotore dell'edizione, se non dell'opera stessa, era stata la singolare figura di pensatore e organizzatore culturale di Ernesto Grassi, a cui Garin era apparso la persona idonea sia per le competenze umanistiche, che per essere subentrato a Gentile come storico della filosofia italiana. Grassi infatti, discepolo a Friburgo di Martin Heidegger, ambiva a mediare fra le scuole filosofiche tedesca e italiana, moderando l'ontologismo di quest'ultimo, di cui pur si faceva portavoce in Italia, con elementi dell'idealismo e spiritualismo italia$\mathrm{ni}$, non senza intenti, peraltro tipici dell'epoca, di emulazione nazionale ${ }^{26}$. Per usare le sue stesse parole, egli aveva mirato a stemperare la negazione nazista dell' «educazione umanistica tradizionale» vantando le iniziative del fascismo per glorificare il Rinascimento italiano, come in particolare la fondazione a Firenze dell'Istituto Nazionale di Studi sul Rinascimento. «Hai visto - scriveva nel 1942 all'amico Enrico Castelli - che Papini ha riferito per le sezioni estere del Centro del Rinascimento?», così attestando lo stretto rapporto fra l'Istituto fiorentino (di cui Papini era presidente), e il 'Centro di studi umanistici e filosofici', che il Grassi aveva fondato a Milano ${ }^{27}$. Ora, proprio nella collana filosofica diretta da Grassi in Svizzera (insieme a W. Szilasi) era uscita la Lettera sull'Umanesimo di Heidegger, in risposta al quesito posto da un accademico francese, J. Beaufret, «Comment redonner un sens au mot 'Humanisme'? ${ }^{28}$. È questa una delle più radicali asserzioni dell'assoluto ontologismo del pensatore tedesco, fino

(25) G. GENTILE, Il carattere del Rinascimento, in Il pensiero italiano, del Rinascimento, cit., p. 17.

(26) Ne hanno trattato recentemente due storici americani; cfr. J. Hankins, Two Twentieth-Century Interpreters of Renaissance Humanism: Eugenio Garin and Paul Oskar Kristeller, ora in ID., Humanism and Platonism in the Italian Renaissance. Vol. I, Humanism, Roma 2003, pp. 573-590, in particolare pp. 609-610; e C. S. CELENZA, The Lost Italian Renaissance. Humanists, Historians, and Latin's Legacy, Baltimore and London 2004, pp. 16-57, in particolare pp. 33-35 (cap. II, Italian Renaissance Humanism in the Twentieth-Century. Eugenio Garin and Paul Oskar Kristeller). Sul Grassi, cfr. P. Donatelli, in Diz. Biogr. Ital., 58 (2002), pp. 607609. Sulle precise circostanze della sollecitazione di Grassi a Garin, da quest'ultimo rivelate in un' $A v$ vertenza alla ristampa del 1994, cfr. M. CAPATI, Cantimori, Contini, Garin, cit., p. 86.

(27) Cfr. M. Simonetta, Filosofia e potere: su Ernesto Grassi, in «Intersezioni», XV (1995), pp. 463 471, qui p. 469. Per l'insofferenza di Croce verso questa e consimili allocuzioni ideologico-retoriche, che avevano come sede deputata «La Rinascita», organo del neo-istituito Istituto di Firenze, si veda la sua lettera a don Giuseppe De Luca, 30 aprile 1939: «La mia critica è rivolta a codesto centro di studi, alla gente che è posta a divulgarlo e alle scemenze che stampano» (cfr. L. MANGONI, In partibus infidelium. Don Giuseppe De Luca: il mondo cattolico e la cultura italiana del Novecento, Torino 1989, p. 289). Merita segnalare qui la figura di amico e corrispondente del Grassi di Enrico Castelli, a lui in qualche modo simmetrica. Esistenzialista cattolico, fu anch'egli fertile promotore di iniziative culturali. Fondatore nel dopoguerra di un 'Centro internazionale di studi umanistici', vi organizzò numerosi convegni, quali ad esempio Umanesimo $e$ machiavellismo (Padova 1949); Umanesimo e scienza politica (Milano 1951); Umanesimo e simbolismo (Padova 1958); Umanesimo e esoterismo (Padova 1960). Castelli, per inciso, fu in precoce rapporto con Garin, per il quale scrisse la Prefazione alla raccolta La disputa delle arti nel Quattrocento (Firenze 1947); mentre a sua volta Garin lo menziona con simpatia nelle Cronache di filosofia, cit., p. 522.

(28) Cfr. C. Celenza, The Lost Italian Renaissance, cit., p. 33. 
al completo ripudio della metafisica razionale classica: «La storia dell'Essere non è né la storia dell'uomo e dell'umanità, né la storia della relazione umana con gli esseri e con l'Essere. La storia dell'Essere è l'Essere stesso e solo l'Essere» ${ }^{29}$. Mentre la tradizione della metafisica greca valeva comunque a offrire un termine di confronto, radicale era invece il rigetto di quella latina, e di qui - nella buona tradizione germanica - del Rinascimento italiano, definito come «renascentia romanitatis», come dire un Umanesimo di terza categoria ${ }^{30}$. A tanto aveva reagito l'eclettismo nazionalistico del Grassi, giustapponendo - non già contrapponendo - alla mai ripudiata dottrina di Heidegger la facoltà di attingere l'insondabile Essere mediante il linguaggio retorico e poetico di scaturigine umanistica e vichiana ${ }^{31}$. E tale, per inciso, fu l'indirizzo che egli diede nel dopoguerra alla sua scuola di Monaco, particolarmente attenta all'Umanesimo italiano. Rhetoric as Philosophy, è il titolo di una recente traduzione inglese dei saggi di Grassi ${ }^{32}$. Rhetorik als Philosophie: Lorenzo Valla, ripeteva una zelante adepta della scuola di Monaco, giungendo ad attribuire all'umanista italiano il concetto heideggeriano del Sosein, dell'essere in sé e per sé, nella dispersione delle esistenze empiriche ${ }^{33}$.

$\mathrm{Fu}$, come si è detto, per controbilanciare tali assunti radicali, che Grassi patrocinò contestualmente il libro di Garin - interprete recente dell'Umanesimo nella storia della filosofia italiana - nella medesima veste linguistica della Lettera di Heidegger, mosso dall'intento, come poi scrisse Garin stesso, «di far convergere due mondi di cultura ${ }^{34}$. Intento del resto non disatteso da Garin, che con il suo 'Umanesimo civile', prevalente ora sulla 'dignità dell'uomo', poneva il suo accento sulla prassi, e, per essa, sul suo strumento espressivo, la retorica politica e morale. E alla glorificazione della 'retorica', spesso indicata allusivamente tra virgolette, a rimarcarne la differenza da una semplice tecnica del discorso letterario, fu dedicata la raccolta già menzionata dei Prosatori latini del Quattrocento, sicuramente la sua più fortunata e influente. E tuttavia la formula, una volta di più, suona come un sottile adattamento di quella celebre gentiliana, dell'Umanesimo come 'filosofia di non filosofi', tradotta in una 'retorica' caricata dei significati più seducenti: «Tutto è, veramente, nel Quattrocento, 'retorica', sol che si ricordi che, d'altra parte, 'retorica' è umanità, ossia spiritualità, consapevolezza, ragione, discorso di uomini»>"

Non s'intende senza queste premesse l'opera di Paul Oskar Kristeller, sicuramente il maggiore studioso dell'Umanesimo del secolo ora concluso, in pari grado per i contributi propri e per l'impulso dato in sede internazionale alla ricerca ${ }^{36}$. Studioso di filosofia di formazione neokantiana - da cui derivò un'indistruttibile fede

(29) Cfr. R. Woun, Heidegger's Children. Hannah Arendt, Karl Löwith, Hans Jonas, and Herbert Marcuse, Princeton-Oxford 2001, pp. 230-231.

(30) Cfr. C. CELENZA, op. cit., p. 34; un'analoga squalifica dell'Umanesimo latino è anche in W. JAEGER, Humanism and Theology (1947); cfr. R. FUBINI, L'Umanesimo italiano, cit., pp. 326-327.

(31) Cfr. P. Donatelli, Grassi, cit., p. 608.

(32) E. Grassi, Rhetoric as Philosophy. The Humanist Tradition, Carbondale, Ill., 2000; cfr. C. Celenza, op. cit., p. 171.

(33) H. B. GERL, Rhetorik als Philosophie: Lorenzo Valla, München 1974, p. 181: «das notwendige Sosein»; per il contesto cfr. R. FuBINI, Umanesimo e secolarizzazione, cit., pp. 359-360.

(34) Cfr. E. GARIN, Cronache di filosofia, cit., p. 515 .

(35) Prosatori latini del Quattrocento, cit.,
(36) Con i contributi di J. Hankins e C. Celenza, si vedano i testi di derivazione autobiografica in Paul Oskar Kristeller and His Contribution to Scholaship, in Philosopby and Humanism. Renaissance Essays in Honor of Paul Oskar Kristeller, ed. by E.P. MAHONEY, Leiden 1976, pp. 1-16; M. KING, Iter Kristellerianum: the European Journey (19051939), in «Renaissance Quarterly», XLVII (1994), pp. 907-909; e inoltre R. FUBINI, L'Umanesimo italiano, cit., pp. 317-332. A parte la monografia sul pensiero del Ficino (Firenze 1953) e, naturalmente, I'Iter italicum, i principali contributi di Kristeller sono ora riuniti in Studies in Renaissance Thought and Letters, 4 voll., Roma 1956-1996; si veda anche, in traduzione italiana, La tradizione classica nel pensiero del Rinascimento, Firenze 1965; Otto pensatori del Rinascimento italiano, Milano-Napoli 1970; Concetti rinascimentali dell'uomo e altri saggi, Firenze 1978. 
nell'autonomia della scienza - ricevette nondimeno un impulso significativo alle sue ricerche dalle personalità, che già abbiamo avuto occasione di nominare, di M. Heidegger e G. Gentile. Già autore di uno studio sull'etica di Plotino, fu incoraggiato da Heidegger, che aveva seguito a Friburgo nel tirocinio post-dottorale, a studiare in Italia l'irradiazione rinascimentale del neo-platonismo con Marsilio Ficino, sul cui pensiero preparò una monografia, uscita solo molto più tardi per le vicissitudini dei tempi. In Italia, presso la Scuola Normale di Pisa, egli godette parimenti del sostegno di G. Gentile: fu appunto nella collana di testi umanistici, allora fondata da Gentile, che Kristeller pubblicò il Supplementum ficinianum, vasta ricerca delle testimonianze manoscritte di Ficino e del suo ambiente, che rinnovava in modo sostanziale la nostra conoscenza della cultura fiorentina di fine Quattrocento ${ }^{37}$. Fu da tale esperienza che Kristeller fu indotto all'esplorazione sistematica di quell'autentico mondo sommerso che era in tutta la sua estensione italiana ed europea l'Umanesimo rinascimentale. Risultato fu il repertorio dell'Iter italicum, che segna una vera data di rifondazione della ricerca sull'Umanesimo ${ }^{38}$. Ma per l'autore l'ingente fatica dell'Iter - incongrua a prima vista per chi faceva professione di filosofia - rappresentò qualcosa di ancor maggiore, e non soltanto strumentale significato. Egli volle con ciò offrire testimonianza di una pura Wissenschaft (esercitata ormai in terra d'America), che si accompagnò altresì a una peculiare interpretazione del fenomeno umanistico. In speculare, seppur perlopiù implicito contrasto con l'attualismo di Gentile (perpetuato nel più cauto e sfumato pragmatismo retorico di Garin), Kristeller intese separare categoricamente il campo designato dagli 'studia humanitatis' (grammatica, retorica, poetica, storia e filosofia morale) da quello propriamente filosofico, da lui essenzialmente identificato nella metafisica razionale (o philosophia perennis) che dall'antica Grecia si estendeva alla Germania di Kant ed Hegel «e oltre», ma comunque circoscritto, come Kristeller tiene a specificare, al «meglio del pensiero moderno» ${ }^{39}$.

L'attenzione rivolta al duplice campo, parallelo e distinto, delle discipline propedeutiche e, d'altra parte, della filosofia propriamente intesa faceva tutt'uno, per Kristeller, con il monito a preservare una continuità di tradizione, entro lo stretto dominio della scuola. Ogni soggettivismo era bandito, e con esso le ideologie, indelebilmente compromesse dalle tragedie del xx secolo. Contro l'ontologismo di Heidegger, enucleato da ogni remora di umana ragione e moralità, era rivendicata, si è detto, la metafisica e teologia classica; contro l'onnicomprensivo attualismo di Gentile era affermato il valore in sé di un'erudizione non importa quanto modesta, e tuttavia tassello prezioso per riallacciare le fila di una tradizione interrotta e tuttora percepita come ulteriormente pericolante. Il nemico da battere era dunque l'ideologia, e per essa la forza disgregatrice delle contrapposizioni ideologiche. Il descrittivismo erudito e l' "oggettiva" esposizione delle dottrine, portata fino alla spersonalizzazione della storia e dei suoi nessi molteplici, acquistavano in ciò la valenza di un'implicita ma effettiva volontà di censura. Una volontà che diviene palese nel discorso pronunciato da Kristeller nell'occasione del conferimento della laurea bonoris causa all'Università di Roma nel 1989: «La novità, l'originalità e la creatività non hanno nessun valore se i loro prodotti non corrispondono alla verità, anzi sono dannose quando portano a

(37) Supplementum ficinianum: Marsilii Ficini Florentini philosophi platonici opuscula inedita et dispersa, voll. 2, Firenze 1937.

(38) P.O. KRISTELLER, Iter italicum. A finding list of uncatalogued or incompletely catalogued bumanistic manuscripts of the Renaissance in Italian and other libraries, voll. 6, Leiden-London 1963-1997.
(39) Cfr. R. Fubini, L'Umanesimo italiano, cit., pp. 328-329. La diffidenza di Kristeller andava particolarmente a Heidegger; come ha testimoniato J. Hankins, suo discepolo, egli in privato si mostrava «unsympathetic to Heidegger as a philosopher, though he admired him as an historian of philosophy»; cfr. J. Hankins, op. cit., p. 583. 
delle asserzioni false e rifiutabili [...]. Dobbiamo pure separare le nostre conoscenze valide, documentabili e oggettive dalle nostre preferenze religiose, politiche e ideologiche» ${ }^{40}$.

$\mathrm{Ma}$ in tal modo - non possiamo esimerci dall'osservare - la benintenzionata 'verità oggettiva' di Kristeller non suona meno astratta, meno lontana dal mondo reale degli uomini, del siderale Essere di Heidegger; e così il paradigma riduttivo dell' 'umanista', in sostanza il didatta dell'antica enkyclios paideia, non suona meno generico di quello anacronistico dell' intellettuale', dedito o meno che fosse alla vita attiva o a quella contemplativa, cultore di uno spiritualismo assoluto alla Pico della Mirandola, o di quell'aspro naturalismo, che Garin giungeva in data tarda a riconoscere in Leon Battista Alberti ${ }^{41}$. Ora un punto è significativo e caratterizzante della fase di studi che abbiamo attraversato. Si tratti di Garin a correttivo dell'idealismo gentiliano, o di Ernesto Grassi a correttivo dell'ontologismo assoluto di Heidegger, o di Kristeller a correttivo dell'ideologismo delle filosofie correnti, tali pur tra sé discordi autori sfociano in una celebrazione della retorica, vale a dire di un discorso che più non sa precisare i propri contenuti. Si tratta in altri termini di filosofie di epigoni, che hanno smarrito un positivo contatto con la storia. Gli esiti, presso prosecutori o discepoli, sono stati più volte aberranti, ai danni, non per caso, del più importante e complesso degli umanisti del XV secolo, Lorenzo Valla. Abbiamo così veduto Valla posto al centro della rivalità eterna di retorica e filosofia ${ }^{42}$; ovvero, addirittura, come il pensatore religioso che con la retorica contribuisce a liberare la teologia cristiana dall'invasiva dialettica aristotelica del Tomismo ${ }^{43}$; mentre, come già si è avuta occasione di dire, una studiosa tedesca sulle tracce di E. Grassi, giungeva a distillare dalla retorica valliana (che supponeva in realtà una filosofia precocemente empirica e utilitaristica) l'oggettività necessitante dell'Essere di Heidegger ${ }^{44}$.

In altri termini, perché riparta la ricerca sull'Umanesimo bisogna rivolgersi altrove che a questi filosofemi di epigoni. La via maestra, ai giorni di Burckhardt (che era al suo tempo un isolato) come al presente è quella di una schietta ricostruzione storica $^{45}$. In tal senso un punto di riferimento hanno offerto due altri studiosi, sia pur reciprocamente distanti, che hanno indirizzato la ricerca sull'Umanesimo italiano nel dopoguerra. Vivaci discussioni ha esercitato al riguardo il libro di Hans Baron, La crisi del primo Rinascimento italiano, uscito in prima edizione in America nel 1955, e tradotto in italiano nel $1970^{46}$. Baron, studioso tedesco che, al pari di Kristeller, apparteneva alla diaspora accademica negli Stati Uniti, fin dagli anni '20, e particolarmente in una silloge di scritti di Leonardo Bruni (1928), aveva insistito sul concetto di 'Umanesimo civile' (o «Bürgerhumanismus»): un Umanesimo cioè, che, contro gli eccessi di individualismo al modo di Burckhardt, si integrasse nelle vecchie buone strutture del Comune cittadino, fino a riconoscersi, di fronte alla minaccia per la libertà rappresentata dal dispotismo egemonico del potere visconteo di Milano, nel repubblicanesimo ideale dell'antica Grecia e di Roma. La tesi, come si è detto, è stata molto influente e

(40) Cfr. R. Fubini, op. cit., p. 330.

(41) Cfr. E. Garin, Rinascite e rivoluzioni. Movimenti culturali dal XIV al XVIII secolo, Roma-Bari 1975, pp. 131-196.

(42) Cfr. J.E. SEIgeL, Rhetoric and Philosophy in Renaissance Humanism. The Unity of Eloquence and Wisdom, Petrarch to Valla, Princeton 1970. Non posso soffermarmi su quanto correttamente sia interpretata, in una tale luce, la stessa retorica antica.

(43) Cfr. S.I. Camporeale, Lorenzo Valla. Umanesimo e teologia, Presentazione di E. GARIN, Firenze 1971.
(44) Cfr. qui sopra, n. 33.

(45) Mi ricollego qui a quanto scritto in R. FUBINI, Renaissance Humanism and its development in Florentine civic culture, in Palgrave advances in Renaissance historiograpby, cit., pp. 118-138.

(46) H. BARON, The Crisis of the Early Italian Renaissance. Civic Humanism and Republican Liberty in an Age of Classicism and Tyranny, voll. 2, Princeton 1955; e Revised one volume edition, with an Epilogue, ivi, 1966; di qui è tratta la traduzione italiana, Firenze 1970. 
discussa (ma per la verità, almeno in sede storica, molto più in America che da noi) ${ }^{47}$. E tuttavia, malgrado l'importante funzione di stimolo, il libro, uscito peraltro troppo tardi e già invecchiato rispetto alla ripresa della ricerca nel dopoguerra, ha finito per denotare le sue gravi manchevolezze, anche sul piano della pur puntigliosa esegesi dei testi umanistici in esame. Meno viceversa si è rilevata la profonda impronta conservatrice, ancora d'impronta germanica, che connetteva la formazione culturale dell'individuo (o 'Bildung') alla pienezza dell'adesione patriottica, nell'ambito della moderna compagine statale. La struttura stessa di The Crisis si ispira direttamente a un saggio di Werner Jaeger (il celebre autore di Paideia), Staat und Kultur del 1932, secondo il quale gli ideali educativi, fin qui individualistici, di Wilhelm von Humboldt avevano trovato la loro perfezione inquadrandosi nei programmi della rinnovata università tedesca e nella corrispondente formazione del buon cittadino ${ }^{48}$.

L'altro studioso a cui ho fatto sopra riferimento è Giuseppe Billanovich, fondatore (o rifondatore, dopo Remigio Sabbadini) della cosiddetta 'filologia medioevale e umanistica', i cui indirizzi furono esemplificati nel periodico da lui fondato nel 1958 , 'Italia medioevale e umanistica'. La filologia di Billanovich, fondatasi principalmente su di un'agguerrita edizione critica dei Rerum memorandarum libri di Petrarca (1945), si affermò poi con un'impronta decisamente ideologica. Era la rivendicazione di parte cattolica di un indirizzo di indagine letteraria fin qui trascurato dalla critica di ispirazione desanctisiana e crociana; referente essenziale per Billanovich fu la figura del suo editore, don Giuseppe De Luca, che parallelamente sulla buona erudizione intendeva fondare una storia del sentimento religioso (o 'della pietà'), scevra di compromissione con le concorrenti istanze modernistiche ${ }^{49}$.

La 'filologia' di Billanovich, che si estendeva nel campo della storia della cultura, e che mantenne Petrarca come principale campo d'attenzione e di orientamento storico, ha avuto insieme uno spiccato merito, e un altrettanto spiccato demerito. Il demerito è quello di aver semplicemente ignorato un'indagine interna ai testi (latini) del Petrarca e ai loro tutt'altro che scontati contenuti, considerandoli al contrario nell'ottica di un generico classicismo, molto debitore della vecchia, buona 'ratio studiorum' di controriformistica memoria. Il merito, cospicuo pur nei suoi limiti, è quello di aver attirato l'attenzione sul tessuto culturale degli scritti petrarcheschi, e sulla loro variegata trasmissione manoscritta ${ }^{50}$. In tal modo il Petrarca letterato (perfettamente coetaneo all'Umanesimo italiano di Garin) ha costituito un'indispensabile pietra di paragone per gli studi petrarcheschi: di qui è nato lo stimolo ad approfondire la questione, ancor oggi tutt'altro che chiara, del rapporto di Petrarca con il suo ambiente, e insieme quella dei tempi e dei modi con cui la sua proposta culturale pervenne agli umanisti del secolo seguente, che da Petrarca attingono molto più di quanto dicano, e in particolare a Lorenzo Valla, delle opere del quale la scuola di Billanovich ha

(47) Cfr. Renaissance Civic Humanism, ed. by J. Hankins, Cambridge 2000. Le tesi di Baron sono al presente influenti soprattutto nella storia delle dottrine politiche, in quanto incorporate nella cosiddetta 'tradizione atlantica', e cioè nel repubblicanesimo di radice inglese e americana; cfr. J.G.A. Pocock, The Machiavellian Moment. Florentine Political Thought and the Atlantic Republican Tradition, Princeton 1975 (trad. ital., Bologna 1980); e Q. SKInNER, The Foundations of modern political thought. Vol. I, The Renaissance; Vol. II, The Reformation, Cambridge 1978 (trad. ital., Le origini del pensiero politico moderno, Bologna 1989).
(48) Cfr. W. JAEger, Humanistische Reden und Vorträge, Zweite erweiterte Auflage, Berlin 1960 , pp. 195-214; e R. FuBINI, Una carriera di storico del Rinascimento, cit., pp. 305-309.

(49) Cfr. L. MANGONI, In partibus infidelium, cit., pp. 304-317; vero e proprio manifesto del nuovo indirizzo fu considerata l'Introduzione di Billanovich al suo Petrarca letterato. I, Lo scrittoio del Petrarca, Roma 1947.

(50) Particolarmente importante al riguardo è G. Billanovich, Le origini dell'Umanesimo. Vol. I, Tradizione e fortuna di Livio tra Medioevo e Umanesimo, Padova 1981. 
promosso alcune edizioni critiche essenziali, fra cui basti nominare le Epistolae, e, più importante ancora, la Repastinatio dialecticae et philosophiae ${ }^{51}$.

Ho accostato ad arte due personalità così distanti come Baron e Billanovich per ragioni, per così dire, autobiografiche. Da ambedue in tempi diversi i miei studi hanno ricevuto orientamento e stimolo, e le conclusioni che ho creduto trarne sono state in qualche modo, per dirla all'inglese, una risposta alla loro sfida.

Per primo viene Billanovich, e cioè Petrarca. La questione del rapporto di Petrarca con il mondo della Scolastica, e in particolar modo con la scolastica avignonese patrocinata dai papi, si è rivelato centrale $e^{52}$. Si tratta infatti, a esaminare nelle loro intenzioni i testi di Petrarca (a cominciare dalle Familiares), colti al di là delle loro dissimulazioni e dei loro accorgimenti retorici, di un rapporto nettamente antagonistico. Non si tratta beninteso delle speculazioni dell'aristotelismo scolastico, ma più ampiamente della Scolastica come metodo che attraversa i secoli, e che riconosce le sue radici nell'età patristica, nell'assunto basilare di trasmettere e commentare i testi di dottrina, dichiarati come 'autorevoli' per sanzione istituzionale ${ }^{53}$. A tale sistema istituzionalizzato di scuola, Petrarca oppone la soggettività umana dei testi, e con essa quella del proprio personale giudizio. E quanto gli permette di interloquire da pari a pari con Agostino ${ }^{54}$, ovvero, in sede storica, di mettere in dubbio tradizioni ricevute, come per esempio nel riesame di Tito Livio. Postulato infine di tutta l'opera petrarchesca è l'equiparazione, dal punto di vista umano, dei testi sacri con quelli profani: il che significava violare il principio gerarchico, che aveva la sua massima sanzione nel De doctrina christiana di S. Agostino. La nozione di 'età oscura' si definisce dal rifiuto di sottostare alla subordinazione utilitaristica del testo profano alle verità della fede: cose fra loro «distantissime», come Petrarca si esprime in un passo dei Rerum memorandarum libri ${ }^{55}$.

Di qui, fin dai primi decenni del nuovo secolo, sarebbero derivate la nuova storiografia politica ${ }^{56}$, una moralistica disincantata e più o meno schiettamente utilitaristica, e con ciò un approccio logico-filosofico, che prendesse le sue mosse, non già dall'esegesi dei testi di tradizione, ma dalla considerazione diretta del reale. Il rappresentante più cospicuo di tale indirizzo, direttamente suggerito - come mette conto ripetere - da Petrarca, è sicuramente Lorenzo Valla (1407-1457). Questi, nel dialogo De vero bono (De voluptate in prima redazione $)^{57}$, rigetta in blocco il tradizionale sistema delle virtù e dei vizi, e considera la moralità dal punto di vista, in sé indivisibile e moralmente neutro, dei moventi verso il piacevole o l'utile (tale è per l'appunto la pseudo-epicurea 'voluptas', che in un primo momento aveva fornito il titolo al dialogo). Sotto un medesimo concetto erano così conglobate le finalità sacre e quelle profane, la Venere mondana e quella celeste: laddove, con l'ontologia del

(51) L. Valla, Repastinatio dialecticae et philosophiae, ed. G. ZIPPEL, Padova 1982; ID., Epistolae, ed. O. Besomi, M. Regoliosi, ivi, 1984.

(52) Cfr. R. FubINI, Intendimenti umanistici e riferimenti patristici dal Petrarca al Valla, in Umanesimo e secolarizzazione, cit., pp. 137-181; e anche ID., Luoghi della memoria ed antiscolasticismo in Petrarca. I "Rerum memorandarum libri", in Visuelle Topoi. Erfindung und tradiertes Wissen in den Künsten der italienischen Renaissance, ed. U. PFISTERER, M. SEIDEL, München-Berlin, 2003, pp. 171-181.

(53) Cfr. R. Fubini, Umanesimo e Scolastica. Saggio per una definizione, in «Medioevo e Rinascimento. Annuario del Dipartimento di Studi sul Me- dioevo e il Rinascimento dell'università di Firenze», XVIII / n.s. XV (2004), pp. 165-174.

(54) Cfr. R. Fubini, Petrarca, S. Agostino e gli Agostiniani, in «Medioevo e Rinascimento», cit., XIX / n.s. XVI (2005), pp. 1-14.

(55) F. Petrarca, Rerum memorandarum libri, Edizione critica a cura di G. Billanovich, Firenze 1945 , p. 29 (I, 25).

(56) Cfr. R. Fubini, Storiografia dell'Umanesimo in Italia, cit., pp. 3-9, 39-51.

(57) Cfr. L. Valla, De vero falsoque bono, ed. M. De Panizza Lorch, Bari 1970. L'intitolazione di quest'edizione, finora insostituita, è in realtà inesatta. 
bene, scompare anche quella del male, e cioè la nozione teologica del peccato. Sicché, a conclusione del dialogo, il personaggio cristiano (quello cioè che estende alla sfera del sacro la nozione della 'voluptas'), esalta al modo di un sermone gli allettamenti del paradiso, mentre tace ad arte delle pene d'inferno ${ }^{58}$. E infine, al pari del Petrarca nella sua considerazione storica e morale, il Valla nella sua opera più propriamente filosofica intende rovesciare l'impostazione che, fin dalle fonti antiche, era stata istituzionalizzata nella scuola: 'repastinatio', come nel titolo della prima edizione, denota l'azione dell'aratro che rovescia la zolla; 'retractatio', secondo i rifacimenti seguenti, indica la volontà di riconsiderare fin dalle fondamenta l" universa philosophia 59 . La 'dialettica' e la 'filosofia' del Valla stanno inseparabilmente a indicare il diretto approccio al reale, contrassegnato nel nome generalissimo della res, 'la cosa', in quanto il solo 'nome' riconoscibile come concetto 'universale', scartando come improprie le 'autorità', vale a dire le concezioni 'probabili' accreditate per tradizione. Ne deriva una netta divaricazione fra dialettica e retorica, sul modello delle verità 'certe' da cui discendono i teoremi della geometria. «Quasi che in filosofia - asserisce il Valla - noi dovessimo imitare i poeti o gli oratori, che frequentemente ricorrono ai 'luoghi' della retorica, vuoi perché così richiedono le circostanze, vuoi per l'ornamento del discorso, vuoi infine per conferirgli una maggiore forza espressiva [...] È questo un discorso del tutto alieno da colui che intenda esprimersi secondo la più rigorosa verità» (procul abest ab eo qui loqui vult ad exactissimam veritatem $)^{60}$.

È questa una visione opposta alla voga corrente, che si è voluto qui sopra illustrare, che nell'esercizio della retorica riconosce il dominio precipuo degli 'umanisti'. La retorica in effetti, non tanto come funzione esornativa, quanto come principio ordinativo del discorso, nonché, più ampiamente, delle tradizioni di dottrina, si sarebbe effettivamente affermata nei curricula scolastici a partire specialmente dalla seconda metà del secolo, trovando la sua maggiore esposizione metodica nel trattato, poi ad ampia diffusione europea, De inventione dialectica di Rodolfo Agricola, olandese ma scolaro in Italia di Battista Guarino, su cui fra gli altri si è soffermato di recente Eckhard Kessler, il più autorevole esponente della scuola di Monaco ispirata da Ernesto Grassi ${ }^{61}$.

Trattando di Umanesimo e Scolastica, quasi dimenticavo di rammentare il mio debito a H. Baron. Pur lontano dalle tematiche metodologiche di cui si è detto, egli ha contribuito in modo, almeno per me, decisivo, a determinare la netta divaricazione fra l'innovativa cultura di Leonardo Bruni a Firenze fin dai primi anni del nuovo secolo, e le tradizioni scolastiche e cancelleresche ancora rappresentate dal suo maestro Coluccio Salutati. L'Umanesimo, anche se solo considerato nel suo stretto aspetto formale, si configurò dunque come un fatto rivoluzionario, come per un improvviso dischiudersi della coltre scolastica, sotto cui stavano annidati i suggerimenti provocatori del Petrarca ${ }^{62}$.

Bruni, che, in un periodo storico di profonde trasformazioni politiche, e in primo luogo di crisi ormai inveterata della Chiesa, aveva saputo conquistarsi i suoi spazi di

(58) Cfr. R. FubINI, Indagine sul "De voluptate" di Lorenzo Valla, in ID., Umanesimo e secolarizzazione, cit., pp. 339-394.

(59) Cfr. L. Valla, Repastinatio dialecticae et philosophiae, cit.; l'intitolazione della redazione ultima è Retractatio totius dialecticae cum fundamentis universae philosophiae.

(60) Cfr. L. VAlla, Repastinatio, cit., p. 389 (I, 9, $\$ 4)$; cfr. R. Fubini, La "Dialectica" di Lorenzo Valla. Saggio di interpretazione, in ID., L'Umanesimo italiano, cit., pp. 184-207.
(61) Cfr. E. Kessler, Renaissance Humanism: The Rbetorical Turn, in Interpretations of Renaissance Humanism, cit., pp. 181-197; ma si veda più ampiamente P. MAcK, Renaissance Argument: Valla and Agricola in the Tradition of Rhetoric and Dialectic, Leiden 1993.

(62) Cfr. R. Fubini, Premesse trecentesche ai "Dialogi ad Petrum Paulum Histrum" di Leonardo Bruni, in «Humanistica. An International Journal of Early Renaissance Studies», 1/2 (2006), pp. 13-21. 
pubblica reputazione e di piena pubblicità dell'opera, rappresentò il più diretto referente per le provocazioni del Valla. Ma con Valla il ciclo si interrompe. Il processo inquisitoriale a Napoli nel 1444, per quanto rimasto interrotto per l'intervento di re Alfonso d'Aragona, ebbe comunque l'effetto di impedire che l'opera filosofica, oggetto dell'imputazione, acquistasse credito e pubblicità, ed egli fu di conseguenza riconosciuto essenzialmente come il precettore grammaticale delle Elegantiae latinae linguae, mentre l'opera di revisione linguistica delle Annotationes in Novum Testamentum acquistò notorietà solo in seguito all'intervento di Erasmo. Fu questa l'epoca in cui i 'literati homines', gli 'eruditi', i 'philosophi', come si definivano sulle tracce di Petrarca, i vari Bruni, Valla, Poggio Bracciolini, Biondo Flavio, Leon Battista Alberti (e come si sarebbero detti i futuri 'hommes de lettres', 'savants', 'philosophes'), ripiegarono sulla più circoscritta professione dell'humanista', l'insegnante di 'umanità', e cioè di un tirocinio di studi preliminare alle professioni universitarie propriamente dette ${ }^{63}$.

Per una tale, determinante svolta epocale, gli esiti più innovativi del primo Umanesimo italiano si prolungarono nei secoli fuori della loro sede primitiva, come si può tangibilmente verificare dalla sede di pubblicazione delle opere. Ciò ha esercitato un impatto anche nella questione tuttora aperta dell'influsso dell'Umanesimo italiano nella cultura europea fino al tempo dell'Illuminismo. Quando notiamo per esempio che Bacone, in The Advancement of Learning, cita implicitamente il proemio all'Italia illustrata di Biondo (a proposito della frammentarietà delle memorie storiche), non va certo a ricercare fra i lasciti manoscritti del XV secolo, ma attinge alla molto più ravvicinata edizione di Basilea degli Opera del 155964; e lo stesso può essere ripetuto per l'esame che Leibniz conduce nei suoi Essais de théodicée del dialogo De libero arbitrio di Lorenzo Valla: di quel Valla di cui egli recuperava la dignità di 'filosofo' («qu'il n'était pas moins Philosophe qu'Humaniste») ${ }^{65}$.

Per tali vicissitudini epocali, legate anche a non sempre visibili pressioni censorie, è improprio parlare di una visione unitaria dell'Umanesimo italiano quattrocentesco. Piuttosto che a una categoria astratta, dobbiamo pensare a processi di cultura, articolati anche sui tempi lunghi, le cui connessioni non sempre sono immediatamente percepibili all'occhio dello storico. Per rispondere a un quesito del prof. Viano al convegno, se si possano stabilire nessi tra 'Umanesimo' e 'Illuminismo', alle condizioni dette darei senz'altro risposta affermativa, nel senso appunto dei tempi lunghi e del processo di durata secolare di affrancamento dalla vecchia Scolastica, che con tanta nettezza già era stato tracciato dai prosecutori ideali del Petrarca nella prima metà del $\mathrm{XV}$ secolo $^{66}$. Sui tempi e sui modi, naturalmente, la ricerca rimane aperta.

RICCARDO FUBINI

(63) Cfr. R. Fubini, "Sogno" e realtà dell'Umanesimo. Contributi recenti sull'Umanesimo italiano, in «Archivio storico italiano», CLX (2002), pp. 87-111; e qui sopra, n. 4. Sui processi di pubblicazione autorizzata nell'epoca del codice manoscritto, cfr. G. Albanese, D. Pietragalia, «In honorem regis edidit». Lo scrittoio di Bartolomeo Facio alla corte napoletana di Alfonso il Magnanimo, in Studi su Bartolomeo Facio, a cura di G. Albanese, Pisa 2000, pp. 1-44.

(64) Cfr. R. Fubini, Storiografia dell'umanesimo in Italia cit, pp. 47-48.

(65) Cfr. E. GARIN, Lorenzo Valla e l'umanesimo, in Lorenzo Valla e l'umanesimo italiano, Atti del convegno internazionale di studi umanistici, a cura di O. Besomi e M. Regoliosi, Padova 1986, pp. 117 , qui p. 3. Si veda al riguardo L. Valla, Dialogue sur le Libre-arbitre, Édition critique, introduction et notes par J. ChOmarat, Paris 1983.

(66) Fra gli storici italiani è rimasto isolato e pressoché sconosciuto il suggerimento di Gaetano Salvemini, che nel suo ultimo corso di lezioni a Firenze nel 1950 così obiettava ad A. Omodeo: «La coltura illuminista è né più né meno che la cultura del Rinascimento». Stroncata in Italia e nei paesi cattolici dalla Controriforma, essa «ha continuato a svilupparsi in Inghilterra, in Francia, in Olanda e nei paesi protestanti della Germania [...] Non esiste soluzione di continuità fra la Rinascenza italianaeuropea dei secoli XIV-XVI e l'illuminismo europeo del secolo XVIII», ecc. (cfr. A. Galante GarRone, Salvemini e Mazzini. In appendice lezioni inedite di Salvemini, Messina-Firenze 1981, p. 495). 